\title{
CERN puts research on hold to build collider
}

Alison Abbott, Munich

CERN, the European particle-physics laboratory in Geneva, Switzerland, is to shelve most of its medium-term research plans in a bid to ensure the completion of its main project - the construction of the Large Hadron Collider (LHC).

Under a retrenchment plan agreed on 21 June between the laboratory's management and its governing council, physicists at CERN will generate practically no fresh experimental data during 2005. Some researchers have already expressed fears about the impact of this data drought on the laboratory.

Cost overruns totalling SFr850 million (US\$570 million) on the LHC's SFr2.6billion construction cost were exposed last autumn (see Nature 413, 557;2001). Council members, whose governments foot CERN's bill, were angry that its director, Luciano Maiani, had known about the problem for months but had not informed them.

In September, Maiani set up internal task forces to consider improvements in CERN's management and to find ways of saving money to pay for completion of the LHC. CERN's council, meanwhile, established an external review committee, chaired by Robert Aymar, director of ITER, the inter-

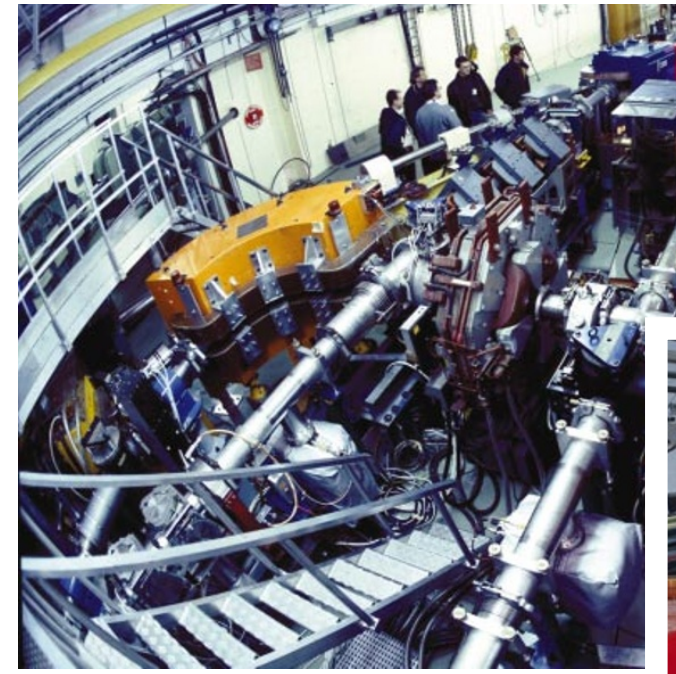

Cost cutting: the Proton Synchrotron (above) and Super Proton Synchrotron will be shut down for all of 2005.

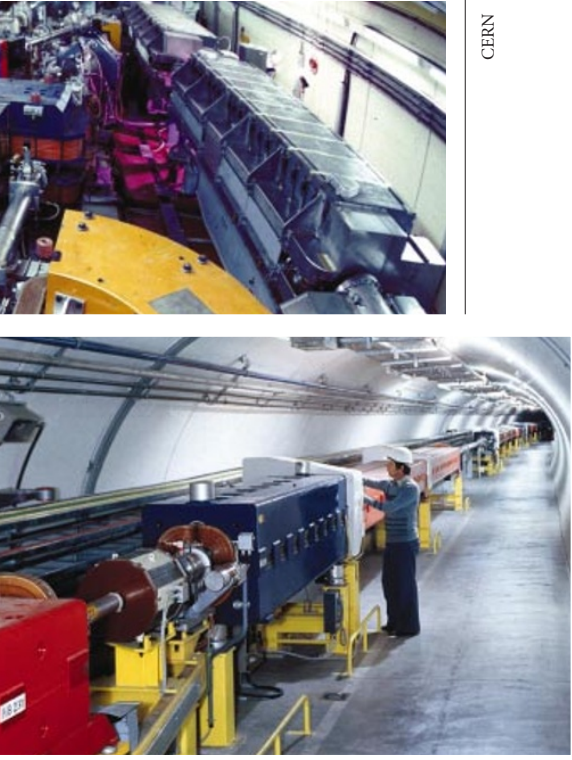

national project to build an experimental magnetic fusion reactor.

Both processes reached similar conclusions, and CERN's council has now accepted Maiani's plan to implement these by streamlining the laboratory's management, winding

\section{Ocean carbon study to quit Hawaii}

Virginia Gewin, Washington

An international consortium is withdrawing its application for a permit to conduct a large carbon-sequestration experiment off the coast of Hawaii, in the face of opposition from environmentalists.

The researchers, who plan to release tonnes of liquefied carbon dioxide in the deep ocean, say there are no environmental grounds for dropping the $\$ 5$ million project. They plan to decamp to Norway, and expect to find out in the next few weeks whether they have permission to do the work there.

Results from the experiment are expected to shed light on the feasibility of carbon sequestration in the oceans as a means of mitigating the build-up of carbon dioxide in the atmosphere, which most researchers think causes global warming.

The time taken to secure a permit "was becoming excessive", says Gerard Nihous, an ocean engineer at the Pacific International Center for High Technology Research in Honolulu, which was due to carry out the study. He says the consortium plans to notify the US Environmental Protection Agency (EPA) this week that it is withdrawing its permit application.

The amount of carbon dioxide to be released in Norway will be less than the 60 tonnes envisaged for release in Hawaii, but it will still be the largest experiment of its type, the researchers say.

The project, which is supported by Japan, the United States and Norway, was conceived in the 1997 meetings that led to the Kyoto Protocol. But local environmental groups claimed that the experiment would acidify Hawaii's fishing grounds (see Nature 401, 315; 1999).

Environmentalists are suspicious of the experiment because they see carbon sequestration as a 'red herring' introduced into the Kyoto negotiations to divert attention from the need to reduce greenhouse-gas emissions. "We're against the study because we think it's part of a larger effort that is looking for a panacea that will encourage more fossil-fuel use," says Jeffrey Mikulina, director of the Hawaiian chapter of the Sierra Club, a large US environmental group.

But an assessment performed for the EPA said that the experiment would have no substantial environmental impact. "If we halt small experiments like this, we're never going to get the answers," says Howard Herzog of the Massachusetts Institute of Technology, one of the study's investigators. down small research projects and adjusting the LHC construction plan.

The LHC, which physicists hope will find the Higgs boson, will not now come on line until 2007, two years later than originally planned. Loans have been arranged to extend the period of payment for the LHC until 2009.

Work not related to the LHC is being cut back to concentrate CERN's resources on the collider. Running time at the Proton Synchrotron and the Super Proton Synchrotron, which provide proton beams for a range of experiments, will be cut by $30 \%$ until 2004, and the machines will be switched off for the whole of 2005, saving SFr34 million.

"The loss of beam time and resources will cause problems for high-energy physicists in Europe and beyond, but we will just have to suffer for a limited number of years," says Maurice Bourquin, a particle physicist who is rector of the University of Geneva and also president of CERN's council.

"Of course it is always painful when data stop flowing," says Maiani. "But it is better to make hard decisions than to cultivate illusions." He adds that there are compensations for scientists not connected with the LHC. The accelerators will be refurbished during the close-down, and the resumption of programmes in 2006 will be guaranteed explicitly in CERN's budget.

But some scientists at CERN are not satisfied. Stephan Paul, a spokesman for the Compass programme that studies the structure and function of hadrons, says that "diversity is required to keep a rounded view of high-energy physics". Until now, diversity was a key feature of CERN's culture. "It's a tragedy that it's being eroded," says Chris Tully, a physicist at Princeton University who works on one of the LHC detectors. 